\title{
Determinants of eating patterns and nutrient intake among adolescent athletes: a systematic review
}

\author{
Matias Noll ${ }^{1,2^{*}}$, Carolina Rodrigues de Mendonça ${ }^{2}$, Lorena Pereira de Souza Rosa ${ }^{2}$ and Erika Aparecida Silveira ${ }^{2^{*}}$
}

\begin{abstract}
Background: This review aims to update the influences of sport modalities, sport performance, and non-exerciserelated determinant, on eating patterns and nutrient intake outcomes among adolescent competitive athletes.

Methods: The PubMed and Scopus databases were searched for the last 20 years. Observational and intervention studies of all languages on eating patterns and nutrient intake in adolescent (10- to 19-year-old) competitive athletes were included. Study quality and risk of bias were assessed using a Downs and Black instrument. Moreover, the Grading of Recommendations, Assessment, Development and Evaluations (GRADE) evidence system was used to assess the strength of the body of evidence.

Results: Regarding outcomes of 21 included articles, $95.2 \%$ of studies focused on nutrient analysis, whereas few of the included articles reported eating patterns. As determinants, most studies analyzed the influences of sport-related $(n=10)$ and demographic factors $(n=8)$, among which only sport modalities were significantly associated with nutrient intake. Age and sex were not significantly associated with nutrient intake. All studies were observational, and most were crosssectional $(n=17)$ and conducted in developed countries such as the United States and European nations. Most studies evaluated boys, and the sport that received the most attention was soccer.
\end{abstract}

Conclusions: Athletes do not adjust their nutrient intake or food choice to the demands of the training load or different training sessions, while sport modalities significantly influenced nutrient intake. Moreover, results concerning demographic determinants were inconsistent and thus remain inconclusive.

Trial registration: Prospero CRD42016043310.

Keywords: Youth, Sport, Nutrition, Eating behavior, Dietary intake, food intake

\section{Background}

The benefits and drawbacks of sports for adolescents have been demonstrated [1-4]. Advantages include substantial evidence of psychological and social benefits $[5,6]$, academic benefits [2], and health benefits [7]. Participation in some sports is associated with iron-deficient anemia [8], high dietary supplement intake [9-11], and sudden death among athletes $[12,13]$. Therefore, researchers have raised concerns about the diet quality of adolescent athletes [14-18].

\footnotetext{
* Correspondence: matiasnoll@yahoo.com.br; erikasil@terra.com.br ${ }^{1}$ Instituto Federal Goiano - Campus Ceres, Rodovia GO-154 - Km 3, Ceres, Goiás, GO 76300-000, Brazil

${ }^{2}$ Postgraduate Program in Health Sciences, Faculty of Medicine, Universidade

Federal de Goiás, Goiás, Brazil
}

However, few studies have investigated eating patterns and nutrient intake $[19,20]$ in adolescent athletes $[18,21,22]$. Consequently, there is no consensus as to whether participation in sports is associated with improved eating patterns - i.e. food choice and frequency or macro- and micronutrient intake [23, 24]. Here, we aimed to systematically review the determinants of eating patterns and nutrient intake among adolescent competitive athletes.

The present review is the first to target adolescent athletes and focused on the following research questions: a) whether sports characteristics (i.e. sport modality and training aspects) are associated with eating patterns and nutrient intake and b) whether demographic, socioeconomic, environmental, psychosocial, and cultural factors 
are determinants of eating patterns and nutrient intake. We further aimed to identify gaps in the literature in this field and priority areas for future research. Such information may be valuable for promoting healthy habits that benefit athletes throughout their lives.

\section{Methods}

\section{Protocol and registration}

This systematic review was registered with the International Prospective Register of Systematic Reviews (PROSPERO) (protocol number: CRD42016043310) [25] and conducted according to the PRISMA (Preferred Reporting Items for Systematic Reviews and Metaanalyses) guidelines [26] for the identification, screening, eligibility, and inclusion of articles.

\section{Search strategy and eligibility criteria}

In August 2016, two independent researchers searched the PubMed and Scopus databases, with no language restriction, for articles published between January 1996 and August 2016. The detailed search strategy is presented as Additional file 1.

We included articles with the following characteristics: (a) a research population consisting of adolescent (10 -19 years old, as defined by the World Health Organization [27]) competitive sports athletes, (b) assessment of eating patterns and/or nutrient intake outcomes and their determinants, and (c) observational or interventional studies. In this context, competitive sport was defined as "a human activity capable of achieving a result requiring physical exertion and/or physical skill which, by its nature and organization, is competitive and is generally accepted as being a sport" [5, 28]. Studies that addressed 'exercise', 'physical activity', 'physical education,' or 'recreation' were not included [5]. Eating patterns were understood as food choices and the frequency of meals and foods; and nutrient intake was defined as macro- and micronutrient intakes and energy intake $[16,29,30]$.

The exclusion criteria were: (a) evaluation of eating disorders; (b) evaluation of supplement intake; (c) studies with incomplete data or review articles; (d) populations that included pregnant and lactating women, hospitalized adolescents, disabled people, or amputees; (e) mixing of athletes with non-athletes, unless the athletes' data were reported separately or could be calculated from the data provided; and (f) mixing of adolescents (10-19 years old) with other ages, unless the adolescents' data were reported separately or could be calculated from the data provided.

\section{Review process}

After executing the search strategy (Fig. 1), duplicate articles were removed. Two reviewers (MN and CRM) then independently screened the titles and abstracts of all articles that were identified in the literature search for inclusion in the systematic review. Disagreement on manuscript inclusion was assessed by concordance analysis (percentage of agreement and the kappa test) and resolved by a third reviewer (LPSR) [31]. The remaining articles were read in full and evaluated to determine their eligibility based on the inclusion and exclusion criteria. Finally, the eligible articles were included in the present systematic review. In addition, the reference lists of included articles were searched to identify additional studies missed by database searches.

\section{Data extraction, quality assessment, and synthesis}

From the selected articles, the following data were extracted: authors, publication year, sample size, sex, age, location, sport modality, study design, presence of a

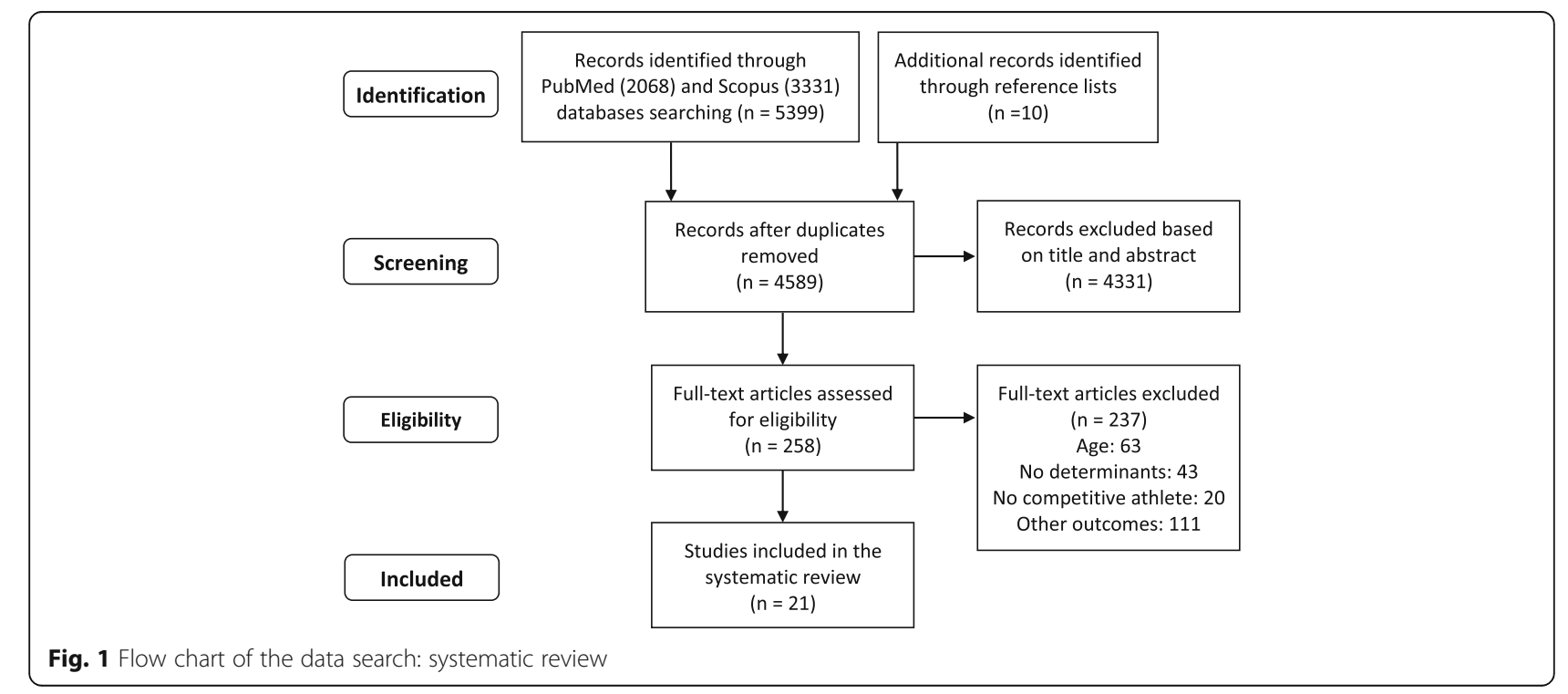


nutritionist in the team of health professionals, outcome, instrument, determinants, and major findings. Study quality and risk of bias were assessed using a 27-item Downs and Black instrument [32]. For observational studies, not all items on the Downs and Black checklist were generally applicable, and a subset of ten questions (Questions 1-3, $6,7,10-12,18$, and 20) was used [33]. A summary quality score for each manuscript was calculated by expressing the number of compliant items as a percentage of the total. Furthermore, the Grading of Recommendations, Assessment, Development and Evaluations (GRADE) evidence system was used to assess the strength of the body of evidence [34]. For each research outcome, quality of evidence was ultimately given one of four grades: high quality, moderate quality, low quality, or very low quality [35].

We also analyzed whether the authors of included studies addressed the impact of possible conflicts of interest and information on ethical approval [36]. Data were extracted and assessed by two independent reviewers ( $\mathrm{MN}$ and $\mathrm{CRM}$ ), and disagreements were resolved by a third reviewer (LPSR). When relevant data were not available in the manuscript, a researcher (MN) contacted the authors directly to fill these gaps.

\section{Results}

\section{Literature search and study selection}

A total of 5399 articles were identified through the PubMed and Scopus database searches, and 10 additional records were identified through reference lists. After removal of duplicates, the titles and abstracts of 4589 records were screened. High concordance $(94.7 \%$ concordance; adjusted kappa $=0.89 ; 95 \%$ CI: 0.88-0.90) [37] between reviewers was found, and 258 articles were selected for full text review (Fig. 1).

After the full text analysis, 21 articles met the eligibility criteria and were included in the present systematic review. Among the 237 excluded studies, the most common reason for exclusion was that they evaluated other outcomes $(n=111)$, followed by not assessing adolescents, not evaluating the determinants of eating patterns and/or nutrient intake, and not evaluating competitive athletes (Fig. 1).

\section{Study characteristics}

All studies were observational in design, and $80.9 \%$ were cross-sectional [38-54] (see Additional file 2). Sample size ranged from 9 to 1138 athletes, with the majority of studies (85.7\%) including less than 100. Most studies evaluated boys [40, 42, 44-46, 49, 50, 52, 55, 56]. Further, $80.9 \%$ studies were conducted in developed countries such as the United States [47, 48, 57] and European nations [38-43, 45, 46, 53, 56, 58, 59]. The sport that received the most attention was soccer $[40,42,44,45,52$, $55,56]$. Only two studies $[41,56]$ reported that the athletes received practical and individual recommendations from a nutritionist.

\section{Outcomes and determinants}

The characteristics of the included studies are shown in Table 1. First, the most common method of evaluation (80.9\%) was the analysis of food records ranging from 1 to 7 days. From these records, 15 studies evaluated three or more days, one study [44] evaluated 2 days, and another study [51] evaluated just 1 day food records. Second, one study used a food weight method with 1 day [46] and three studies used a food weight method with 5 days or more $[39,42,52]$. Third, four studies used a validated food frequency questionnaire $[42,43,51]$ and one [41] used a questionnaire specifically validated for that study. Fourth, one study used a food diary associated with food records [54].

The 21 articles were analyzed and categorized based on their assessment of two broad outcomes: food and nutrient intake. Only four studies presented results regarding food intake [41-43, 58]. As determinants for these outcomes, most studies analyzed the influences of sport-related factors $(n=10)$, age $(n=3)$, sex $(n=6)$, and Ramadan $(n=2)$. Other rarely evaluated determinants included food preference, menu settings, meal patterns, place of eating, and type of school (Table 1). The determinants to both outcomes are described below:

\section{a) Sports training and modality}

Ten studies evaluated the influence of sport-related factors on food and nutrient intake. Five studies found that athletes did not adjust their nutrient intake to the demands of different training loads and sessions. Briggs et al. [39] compared four training days (heavy and moderate training, match, and rest) and found no differences in macronutrient intake. Caccialanza et al. [40] performed two evaluations separated by a 3-month period and also found no significant variations in reported energy, macronutrient, fiber, or cholesterol intakes between evaluations. Papadopoulou et al. [53] compared two training categories of volleyball players and found no differences in macro- or micronutrient intakes with the exception of fat ingestion. Kabasakalis et al. [59] and Ziegler et al. [57] reported no significant differences in macronutrient intakes in 8- and 9-month studies, respectively. Similarly, Boisseau et al. [38] found no differences in micro- and macronutrient intake across weeks of a training period with the exception of lipid ingestion and carbohydrate. However, Thivel et al. [46] observed differences in meal composition in a study comparing different training sessions (control session, rugby session, and exercise session) (Table 2). 


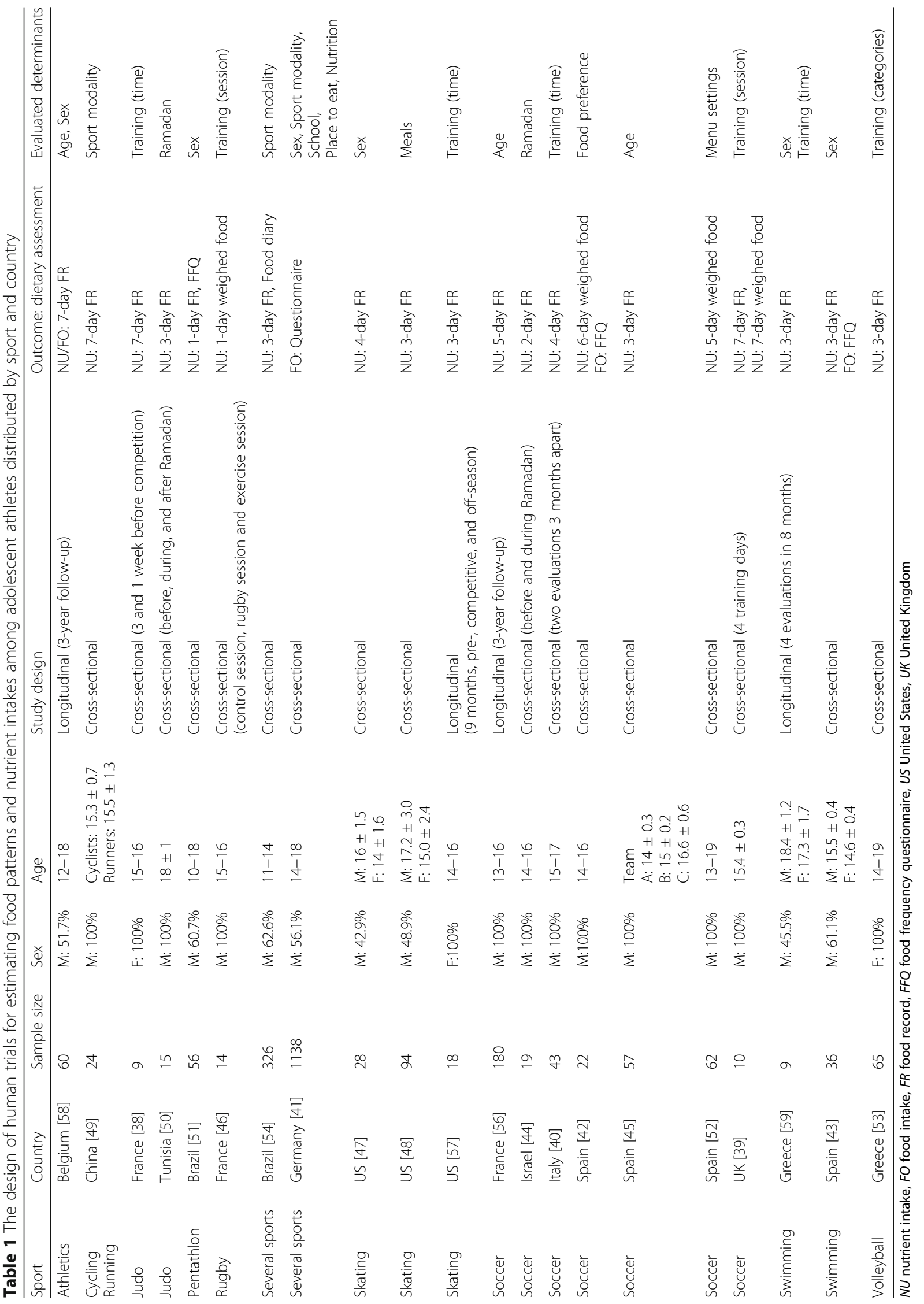




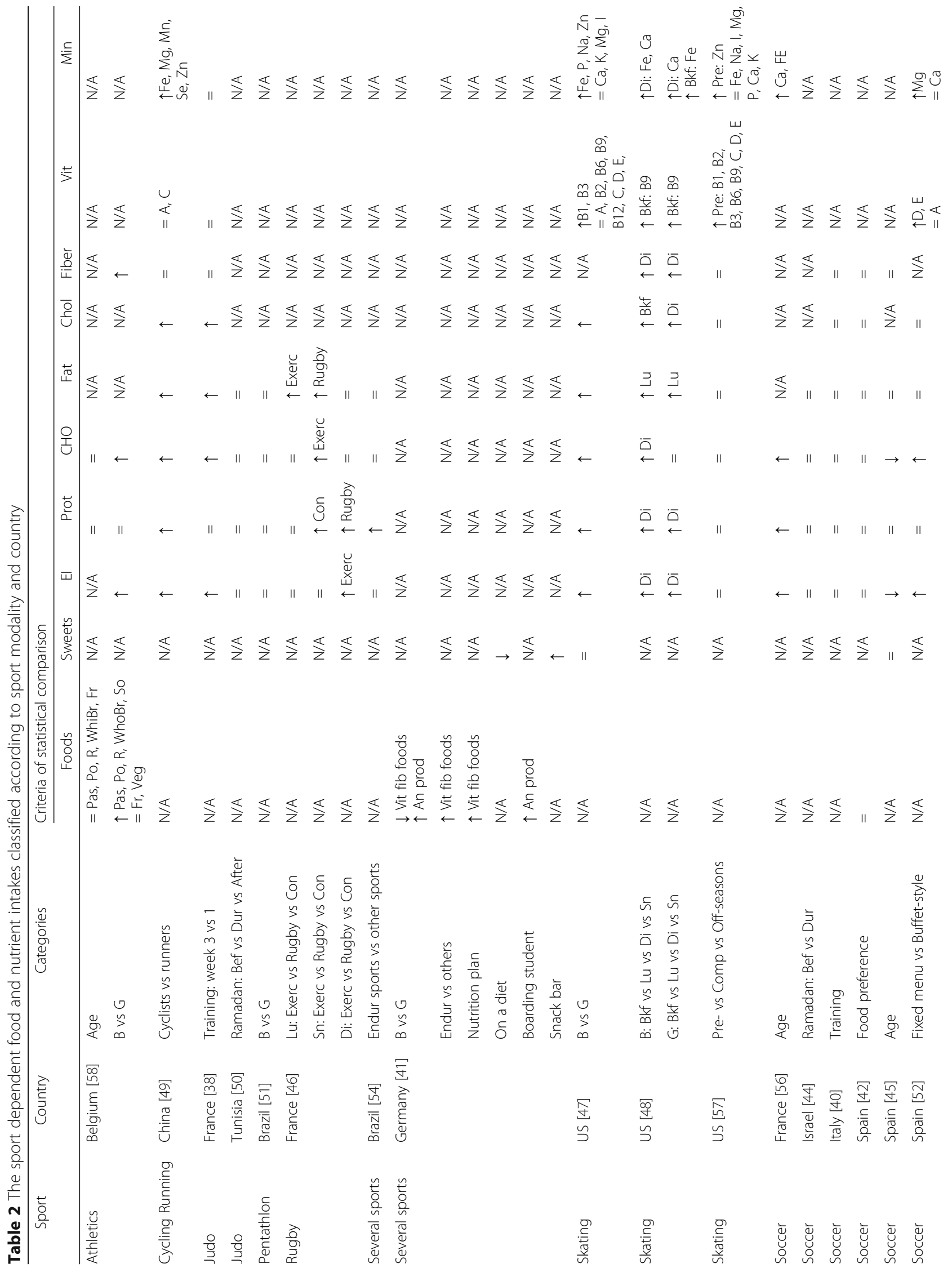




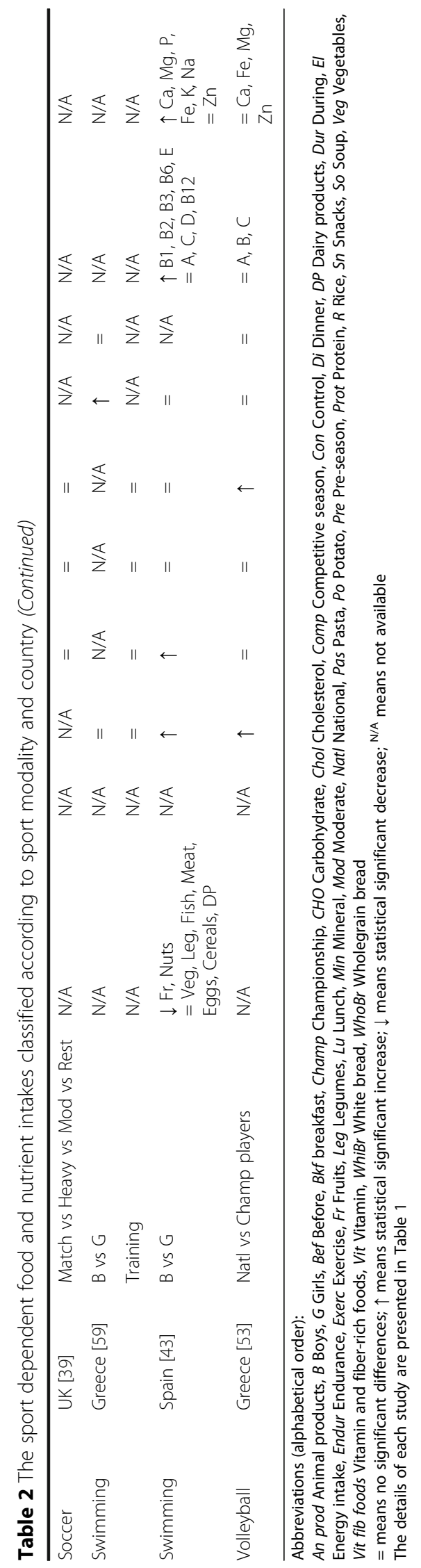


Three studies verified the association between sport modalities and food and nutrient intake. Diehl et al. [41] found that endurance sport athletes eat more foods high in vitamins and fiber than do competitors in strength sports, ball games, or aesthetic sports. Sousa et al. [54] also evaluated several sports and found differences in protein intake. Tong et al. [49] evaluated runners and cyclists and found that intakes of macronutrients as well as micronutrients were higher in cyclists.

\section{b) Demographic determinants}

Conflicting results were reported concerning demographic (sex and age) determinants. Diehl et al. [41] found better food intake in female athletes, such as consumption of more products high in vitamins and fiber and fewer animal products. Martínez et al. [43] also found higher fruit intake among female athletes. However, Aerenhouts et al. [58] found no sex differences in fruit and vegetable consumption, although boys ate more wholegrain bread, pasta, rice, and potatoes than girls. Regarding nutrient intake, Ziegler et al. [47] demonstrated that male athletes had higher total energy and macro- and micronutrient intakes than girls did, and Kabasakalis et al. [59] found higher cholesterol intakes among males. Similar results were found by Martínez et al. [43], who observed higher intakes of macro- and micronutrients among males athletes. By contrast, Coutinho et al. [51], found no differences in energy or nutrient intake between the sexes. In short, the reported role of sex as a determinant for both food and nutrient intake outcomes is inconsistent. These conflicting results could be due to the different methods used; for example, Coutinho et al. [51] evaluated just oneday food records (Table 2 ).

Regarding age influences, Ruiz et al. [45] evaluated three different age groups and carbohydrate intake was found to decrease with increasing age, in parallel with a decrease in the percentage of total energy ingested at breakfast, morning break, and afternoon break. Leblanc et al. [56] found that energy, macro-, and micronutrient intakes increased significantly over a 3-year follow-up period. In another 3-year follow-up study, Aerenhouts et al. [58] demonstrated no differences between ages with regard to nutrient intake and eating patterns. Therefore, results concerning the role of age as a determinant for both food and nutrient intake outcomes are also inconclusive.

\section{c) Others determinants}

The relation between Ramadan and nutrient intake were investigated by two studies [44, 50]; no significant changes on macro- and micronutrient intake before, during, and after Ramadan were found. Other determinants, although evaluated by single studies, were also found to be important. Ziegler et al. [48] examined the contribution of meal patterns (breakfast, lunch, dinner, and snack) on microand macronutrient and found differences between the predominant sources of fat, cholesterol, and dietary fiber intake at lunch and dinner for girls and boys. Garrido et al. [52] evaluated menu settings and found that fixed 'menu-style' menus have more total energy, cholesterol, and micronutrient intakes than do flexible 'buffet-style' menus. One study [42] demonstrated that food preference does not interfere with nutrient intake or the daily number of food portions consumed. Regarding eating patterns, Diehl et al. [41] reported several results: athletes who had a nutrition plan and information from a nutritionist ate more products high in vitamins and fiber; eating meals at the school cafeteria was associated with increased consumption of animal products; and eating at a snack bar and on the way were associated with increased consumption of sweets and snacks (Table 2).

\section{Data quality assessment and strength of evidence}

The Downs and Black checklist scores ranged from 60\% to $100 \%$ (see Additional file 3). Principal risks of bias arose from the lack of reporting of probability values, targeted samples, and recruitment of a representative population. Only two studies $[41,54]$ evaluated a nationally representative sample. Strength of evidence classification using the GRADE methodology indicated that 12 studies were of very low quality. Only the studies by Diehl et al. [41] and Sousa et al. [54] presented a moderate strength of evidence. Notably, just one-fourth of studies $[39,41,46,51,54]$ clearly stated that there were no conflicts of interest, and most studies $(n=13)$ reported ethical approval (see Additional file 3).

\section{Discussion}

Our study is the first to systematically review the determinants of eating patterns and nutrient intake among adolescent competitive athletes. Regarding outcomes, most studies focused on nutrient analysis, and few of the included articles reported results concerning eating patterns. Our findings suggest that athletes did not adjust their nutrient intake to the demands of the training load and different training sessions. However, sport modalities significantly influenced their nutrient intake and eating patterns. Such evidence is valuable to improve diet quality that benefits athletes throughout their lives. Moreover, demographic determinants (age and sex) demonstrated conflicting results, and the cultural determinant of Ramadan did not influence nutrient intake.

We were unable to establish a strong relationship between eating patterns or nutrient intake and environmental (fixed menu [52], eating in a cafeteria or snack bar [41]) or psychosocial determinants (food preference 
[42]; meal patterns [48], information from a nutritionist, nutritional plan, maintenance on a diet [41]) because these variables were each evaluated in only one study. Moreover, we did not identify any studies that evaluated socioeconomic characteristics as possible determinants of eating patterns or nutrient intake.

Several reviews focused on other populations have identified many factors that can influence diet quality. In a nonsystematic review, Birkenhead and Slater [23] highlighted the multidimensional nature of food choices and indicated that nutritional knowledge and physiological, social, and economic factors were associated with food choices. Scaglioni et al. [60] described how eating patterns involve a complex interaction of genetic, familial, and environmental factors. Furthermore, studies focused on non-athlete students [61-63] identified others important determinants including parental control, cultural traditions, climatic factors, and smoking and drinking. Thus, compared to youth [64] and adults [65], there is a lack of evidence concerning the evaluation of determinants for adolescent athletes.

Regarding the two broad outcomes, few studies reported on eating patterns $[41-43,58]$. Until recently, the relationship between eating patterns and health was underestimated, and most guidelines treated foods as mere nutrient carriers [22, 66, 67]. However, current investigations have extended beyond the simple intake of nutrients to consider food choice as well as the level of processing to which foods are subjected $[68,69]$. Data concerning eating patterns are essential $[20,70]$, because nutrition-specific education programs may impact training and performance and should target not only nutrients, but also both food choice and eating habits [22, 48]. Erdman et al. [71] and Iglesias et al. [21] suggested that research on eating patterns and food sources is indispensable because it may improve athletes' knowledge, which is essential to effectively advise them with tangible data [4].

The present systematic review revealed several notable gaps in the literature in this field. First, no study investigated athletes from South Africa, Central America, or Oceania, and most were conducted in developed countries. Second, male athletes were the focus of more studies. Third, most studies were cross-sectional and evaluated small samples. Fourth, soccer was the most commonly investigated sport, while other sports received less attention. Fifth, due the wide range of aims, measurement tools, outcomes, and determinants assessed, a meta-analysis cannot be carried out. Sixth, food records and food weight protocols including less than 3 days are not representative to calculate nutrient intake. Furthermore, other possibly important determinants were not investigated, such as family educational and socioeconomic level, parental rules, coach's knowledge of nutrition and rules, athlete's nutritional knowledge, sponsorship and commercial activities, presence of a nutritionist in the sport team, smoking and drinking, sedentary patterns, and urban or rural residence. These other possible determinants should be considered in future studies [70, 72, 73].

The strength of evidence was low, and the analysis of the methodological quality of the studies indicated that the principal risk of bias was that the sample was not representative of the population. However, convenience samples are common in epidemiological studies [74]. Moreover, we observed that most articles did not contain a clear statement regarding conflict of interest, and this consideration should be taken into account in future studies. This issue has been cited as a concern of the Pan American Health Organization, which has warned of the dangers of conflicts of interest related to nutrition [75]. The World Health Organization addressed this issue in 2015 [76].

Overall, based on the gap in knowledge on eating patterns, the limited number of determinants analyzed, and the results regarding risk of bias and quality of evidence, our results highlight some interesting opportunities for future research, particularly the performance of longitudinal and experimental studies; greater research efforts in developing countries; evaluation of representative samples, preferably including both sexes; assessment of a wide variety of sports, preferably within the same study to allow for comparisons between them; and inclusion of a wider range of determinants. The greater promotion of adequate nutrition during adolescence may optimize training performance as well as improve nutritional knowledge and healthy habits that may benefit the athletes beyond the end of their sports careers [22, 24, 41, 77]. Clubs and sporting organizations may provide an ideal outlet to introduce health-related policies, and preferably with a nutritionist in the team of health professionals [78]. New studies may contribute to the development of preventive programs and health strategies and policies aimed at adolescent athletes $[4,79,80]$ as well as further promote greater collaboration between researchers and research groups through multi-center studies.

Strengths of this study include the identification of specific determinants and the focus on an existing gap in knowledge related to the evaluation of eating patterns among adolescent competitive athletes. Furthermore, this review has several methodological strengths, including the absence of a language restriction; high concordance between reviewers in title and abstract screening; inclusion of study quality and risk of bias analysis; and analyses of conflicts of interest and ethical approval, which are often omitted from reviews.

\section{Conclusion}

In summary, athletes do not adjust their nutrient intake to the demands of the training load or different training 
sessions, while sport modalities significantly influenced nutrient intake. Moreover, demographic determinants are inconsistent and remain inconclusive. Lastly, nutrient intake has received more attention than eating patterns in studies of adolescent athletes. Furthermore, future research should be developed to improve the quality of evidence regarding determinants of eating patterns and nutrient intake.

\section{Additional files}

Additional file 1: Search strategy. The PubMed search terms were: ()(()(()(()(()((food choice[Title/Abstract]) OR food intake[Title/Abstract]) OR food consumption[Title/Abstract]) OR eating behavior[MeSH Terms]) OR nutrition assessment[MeSH Terms]) OR food preference[MeSH Terms]) OR health behavior[MeSH Terms]) OR food habits[MeSH Terms]) OR diet, food, and nutrition[MeSH Terms]) OR nutritional status[MeSH Terms]) OR feeding behavior[MeSH Terms]) OR eating[MeSH Terms]) OR food and beverages[MeSH Terms]) OR diet[MeSH Terms]) OR food[MeSH Terms]) AND (((adolescent[MeSH Terms]) OR students[MeSH Terms]) OR minors[MeSH Terms])) AND ((athletes[MeSH Terms]) OR sports[MeSH Terms]). This search strategy was adapted for the Scopus database. (DOCX $10 \mathrm{~kb}$ )

Additional file 2: Quantitative characteristics of the 21 articles included in the systematic review. (DOCX $13 \mathrm{~kb}$ )

Additional file 3: Methodological quality assessment and strength of evidence. (DOCX $2818 \mathrm{~kb}$ )

\section{Abbreviations}

GRADE: Grading of recommendations, assessment, development and evaluations; PRISMA: Preferred reporting items for systematic reviews and meta-analyses; PROSPERO: International Prospective Register of Systematic Reviews

\section{Acknowledgements}

The authors are grateful to the FAPEG Foundation for scholarships. The authors are also grateful to the Instituto Federal Goiano and GEOG (Study Group on Severe Obesity) for financial fees support.

\section{Funding}

Not applicable.

\section{Availability of data and materials}

Not applicable.

\section{Authors' contributions}

MN: Definition of objectives, development of search strategy, data extraction, data analysis, writing and revision of the manuscript. CRM: Development of search strategy, data extraction, data analysis, writing and revision of the manuscript. LPSR: Approval of search strategy, analysis of discordance, writing and revision of the manuscript. EAS: Definition of the objectives, approval of search strategy, analysis of discordance, writing and revision of the manuscript. All authors read and approved the final manuscript.

\section{Ethics approval and consent to participate}

Not applicable.

\section{Consent for publication}

Not applicable.

\section{Competing interests}

The authors declare that they have no competing interests.

\section{Publisher's Note}

Springer Nature remains neutral with regard to jurisdictional claims in published maps and institutional affiliations.
Received: 24 April 2017 Accepted: 21 July 2017

Published online: 28 July 2017

\section{References}

1. Diehl K, Thiel A, Zipfel S, Mayer J, Litaker DG, Schneider S. How Healthy is the Behavior of Young Athletes? A Systematic Literature Review and MetaAnalyses. J Sport Sci Med. 2012:11:201-20.

2. Bailey R. Physical Education and Sport in Schools: A Review of Benefits and Outcomes. J Sch Health. 2006;76:397-401. doi:10.1111/j.1746-1561.2006. 00132.x

3. Sønderlund AL, O'Brien K, Kremer P, Rowland B, De Groot F, Staiger $P$, et al. The association between sports participation, alcohol use and aggression and violence: A systematic review. J Sci Med Sport Sports Med Aust. 2014; 17:2-7. doi:10.1016/j.jsams.2013.03.011.

4. Bergeron MF, Mountjoy M, Armstrong N, Chia M, Côté J, Emery CA, et al. International Olympic Committee consensus statement on youth athletic development. Br J Sports Med. 2015;49:843-51. doi:10.1136/bjsports-2015094962

5. Eime RM, Young JA, Harvey JT, Charity MJ, Payne WR. A systematic review of the psychological and social benefits of participation in sport for children and adolescents: Informing development of a conceptual model of health through sport. Int J Behav Nutr Phys Act. 2013;10:98-119. doi:10.1186/14795868-10-98

6. Snyder AR, Martinez JC, Bay RC, Parsons JT, Sauers EL, Valovich McLeod TC. Health-related quality of life differs between adolescent athletes and adolescent nonathletes. J Sport Rehabil. 2010:19:237-48.

7. Janssen I, LeBlanc AG. Systematic review of the health benefits of physical activity and fitness in school-aged children and youth. Int J Behav Nutr Phys Act. 2010;7:40. doi:10.1186/1479-5868-7-40.

8. Parnell JA, Wiens KP, Erdman KA. Dietary Intakes and Supplement Use in Pre-Adolescent and Adolescent Canadian Athletes. Nutrients. 2016;8:1-13. doi:10.3390/nu8090526.

9. Knapik JJ, Steelman RA, Hoedebecke SS, Austin KG, Farina EK, Lieberman HR. Prevalence of Dietary Supplement Use by Athletes: Systematic Review and Meta-Analysis. Sport Med. 2016:46:103-23. doi:10.1007/s40279-015-0387-7.

10. Sousa M, Fernandes MJ, Soares J, Moreira P, Teixeira VH. Nutritional supplement-usage associated characteristics of high-performing athletes. $\mathrm{Br}$ Food J. 2016;118:26-39. doi:10.1108/BFJ-03-2015-0088.

11. Petroczi A, Naughton DP, Mazanov J, Holloway A, Bingham J. Limited agreement exists between rationale and practice in athletes' supplement use for maintenance of health: a retrospective study. Nutr J England. 2007;6: 34. doi:10.1186/1475-2891-6-34.

12. Corrado D, Basso C, Rizzoli G, Schiavon M, Thiene G. Does sports activity enhance the risk of sudden death in adolescents and young adults? J Am Coll Cardiol. 2003;42:1959-63. doi:10.1016/j.jacc.2003.03.002.

13. Thiene G, Carturan E, Corrado D, Basso C. Prevention of sudden cardiac death in the young and in athletes: dream or reality? Cardiovasc Pathol. 2010;19:207-17. doi:10.1016/j.carpath.2009.04.001.

14. Parks RB, Helwig D, Dettmann J, Taggart T, Woodruff B, Horsfall K, et al. Developing a Performance Nutrition Curriculum for Collegiate Athletics. J Nutr Educ Behav. 2016. doi:10.1016/j.jneb.2016.03.002.

15. Vella S a, Cliff DP, Okely AD, Scully ML, Morley BC. Associations between sports participation, adiposity and obesity-related health behaviors in Australian adolescents. Int J Behav Nutr Phys Act. 2013;10:113. doi:10.1186/ 1479-5868-10-113.

16. Croll JK, Neumark-Sztainer D, Story M, Wall M, Perry C, Harnack L. Adolescents Involved in Weight-Related and Power Team Sports Have Better Eating Patterns and Nutrient Intakes than Non-Sport-Involved Adolescents. J Am Diet Assoc. 2006;106:709-17. doi:10.1016/j.jada.2006.02. 010.

17. Nascimento M, Silva D, Ribeiro S, Nunes M, Almeida M, Mendes-Netto R. Effect of a Nutritional Intervention in Athlete's Body Composition, Eating Behaviour and Nutritional Knowledge: A Comparison between Adults and Adolescents. Nutrients. 2016:8:535. doi:10.3390/nu8090535.

18. Tawfik S, El Koofy N, Moawad EMI. Patterns of nutrition and dietary supplements use in young Egyptian athletes: A community-based crosssectional survey. Tauler P, editor. PLoS One. 2016:11:e0161252. doi:10.1371/ journal.pone.0161252.

19. Purcell LK. Sport nutrition for young athletes. Paediatr Child Heal. 2013;18:200-2. 
20. Leech RM, Worsley A, Timperio A, McNaughton SA. Characterizing eating patterns: a comparison of eating occasion definitions. Am J Clin Nutr. 2015; 102:1229-37. doi:10.3945/ajcn.115.114660.

21. Iglesias-Gutiérrez E, García Á, García-Zapico P, Pérez-Landaluce J, Patterson ÁM, García-Rovés PM. Is there a relationship between the playing position of soccer players and their food and macronutrient intake? Appl Physiol Nutr Metab. 2012;37:225-32. doi:10.1139/h11-152.

22. García-Rovés P, García-Zapico P, Patterson Á, Iglesias-Gutiérrez E. Nutrient Intake and Food Habits of Soccer Players: Analyzing the Correlates of Eating Practice. Nutrients. 2014;6:2697-717. doi:10.3390/nu6072697.

23. Birkenhead KL, Slater G. A Review of Factors Influencing Athletes' Food Choices. Sport Med. 2015;45:1511-22. doi:10.1007/s40279-015-0372-1.

24. Spronk I, Heaney SE, Prvan T, O'Connor HT. Relationship between General Nutrition Knowledge and Dietary Quality in Elite Athletes. Int J Sport Nutr Exerc Metab. 2015:25:243-51. doi:10.1123/ijsnem.2014-0034

25. Booth A, Clarke M, Dooley G, Ghersi D, Moher D, Petticrew M, et al. The nuts and bolts of PROSPERO: an international prospective register of systematic reviews. Syst Rev. 2012;1:2. doi:10.1186/2046-4053-1-2.

26. Moher D, Shamseer L, Clarke M, Ghersi D, Liberati A, Petticrew M, et al. Preferred reporting items for systematic review and meta-analysis protocols (PRISMA-P) 2015 statement. Syst Rev. 2015;4:1. doi:10.1186/2046-4053-4-1.

27. WHO. Maternal, newborn, child and adolescent health [Internet]. 2016 [cited 2016 Jul 20].

28. The Australian Sports Commission (ASC). Australian Sports Commission: What is defined as a sport [Internet]. [cited 2016 Oct 20].

29. Shriver LH, Betts NM, Wollenberg G. Dietary Intakes and Eating Habits of College Athletes: Are Female College Athletes Following the Current Sports Nutrition Standards? J Am Coll Health United States. 2013;61:10-6. doi:10. 1080/07448481.2012.747526.

30. Nogueira JAD, Da Costa THM. Nutrient intake and eating habits of triathletes on a Brazilian diet. Int J Sport Nutr Exerc Metab. 2004;14:684-97.

31. Stefani L, Galanti G, Padulo J, Bragazzi NL, Maffulli N. Sexual Activity before Sports Competition: A Systematic Review. Front Physiol. 2016;7 doi:10.3389/ fphys.2016.00246.

32. Downs SH, Black N. The feasibility of creating a checklist for the assessment of the methodological quality both of randomised and non-randomised studies of health care interventions. J Epidemiol Community Heal. 1998;52: 377-84. doi:10.1136/jech.52.6.377.

33. Muthuri SK, Francis CE, Wachira LJM, LeBlanc AG, Sampson M, Onywera VO, et al. Evidence of an overweight/obesity transition among school-aged children and youth in Sub-Saharan Africa: A systematic review. PLoS One. 2014;9. doi:10.1371/journal.pone.0092846.

34. Guyatt G, Oxman AD, Akl EA, Kunz R, Vist G, Brozek J, et al. GRADE guidelines: 1. Introduction - GRADE evidence profiles and summary of findings tables. J Clin Epidemiol. 2011;64:383-94. doi:10.1016/j.jclinepi.2010. 04.026.

35. Balshem $H$, Helfand $M$, Schünemann HJ, Oxman AD, Kunz R, Brozek J, et al. GRADE guidelines: 3. Rating the quality of evidence. J Clin Epidemiol. 2011; 64:401-6. doi:10.1016/j.jclinepi.2010.07.015.

36. Elia N, von Elm E, Chatagner A, Pöpping DM, Tramèr MR. How do authors of systematic reviews deal with research malpractice and misconduct in original studies? A cross-sectional analysis of systematic reviews and survey of their authors BMJ Open. 2016:6:e010442. doi:10.1136/bmjopen-2015-010442.

37. Sim J, Wright CC. The kappa statistic in reliability studies: use, interpretation, and sample size requirements. Phys Ther. 2005;85:257-68.

38. Boisseau N, Vera-Perez S, Poortmans J. Food and fluid intake in adolescent female judo athletes before competition. Pediatr Exerc Sci. 2005;17:62-71.

39. Briggs MA, Cockburn E, Rumbold PLS, Rae G, Stevenson EJ, Russell M. Assessment of energy intake and energy expenditure of male adolescent academy-level soccer players during a competitive week. Nutrients. 2015;7: 8392-401. doi:10.3390/nu7105400.

40. Caccialanza R, Cameletti B, Cavallaro G. Nutritional intake of young Italian high-level soccer players: Under-reporting is the essential outcome. J Sport Sci Med. 2007:6:538-42.

41. Diehl K, Yarmoliuk T, Mayer J, Zipfel S, Schnell A, Thiel A, et al. Eating Patterns of Elite Adolescent Athletes: Results of a Cross-Sectional Study of 51 Olympic Sports. Dtsch Z Sportmed. 2013;2013:126-31. doi:10.5960/dzsm. 2012.067.

42. Iglesias-Gutiérrez E, García-Rovés PM, García A, Patterson AM. Food preferences do not influence adolescent high-level athletes' dietary intake. Appetite. 2008;50:536-43. doi:10.1016/j.appet.2007.11.003.
43. Martínez S, Pasquarelli BN, Romaguera D, Arasa C, Tauler P, Aguiló A. Anthropometric Characteristics and Nutritional Profile of Young Amateur Swimmers. J Strength Cond Res. 2011;25:1126-33. doi:10.1519/JSC. Ob013e3181d4d3df

44. Meckel Y, Ismaeel A, Eliakim A. The effect of the Ramadan fast on physical performance and dietary habits in adolescent soccer players. Eur J Appl Physiol. 2008;102:651-7. doi:10.1007/s00421-007-0633-2.

45. Ruiz F, Irazusta A, Gil S, Irazusta J, Casis L, Gil J. Nutritional intake in soccer players of different ages. J Sports Sci. 2005;23:235-42. doi:10.1080/ 02640410410001730160 .

46. Thivel D, Maso F, Aouiche S, Coignet B, Doré E, Duché P. Nutritional responses to acute training sessions in young elite rugby players. Appetite. 2015:84:316-21. doi:10.1016/j.appet.2014.10.030.

47. Ziegler PJ, Khoo CS, Kris-Etherton PM, Jonnalagadda SS, Sherr B, Nelson JA. Nutritional status of nationally ranked junior US figure skaters. J Am Diet Assoc. 1998;98:809-11. doi:10.1016/S0002-8223(98)00181-3.

48. Ziegler PJ, Jonnalagadda SS, Nelson JA, Lawrence C, Baciak B. Contribution of meals and snacks to nutrient intake of male and female elite figure skaters during peak competitive season. J Am Coll Nutr. 2002;21:114-9.

49. Tong TK, Lin H, Lippi G, Nie J, Tian Y. Serum Oxidant and Antioxidant Status in Adolescents Undergoing Professional Endurance Sports Training. Oxidative Med Cell Longev. 2012;2012:1-7. doi:10.1155/2012/741239.

50. Chaouachi A, Chamari K, Roky R, Wong P, Mbazaa A, Bartagi Z, et al. Lipid Profiles of Judo Athletes during Ramadan. Int J Sports Med. 2008;29:282-8. doi:10.1055/s-2007-965338.

51. Coutinho LAA, Porto CPM, Pierucci APTR. Critical evaluation of food intake and energy balance in young modern pentathlon athletes: a cross-sectional study. J Int Soc Sport Nutr. 2016;13:15. doi:10.1186/s12970-016-0127-x.

52. Garrido G, Webster AL, Chamorro M. Nutritional adequacy of different menu settings in elite Spanish adolescent soccer players. Int. J. Sport Nutr. Exerc. Metab. 2007;17:421-32.

53. Papadopoulou SK, Papadopoulou SD, Gallos GK. Macro- and micro-nutrient intake of adolescent Greek female volleyball players. Int. J. Sport Nutr. Exerc. Metab. 2002:12:73-80

54. de Sousa EF, Da Costa THM, Nogueira JAD, Vivaldi LJ. Assessment of nutrient and water intake among adolescents from sports federations in the Federal District. Brazil Br J Nutr. 2008;99:1275-83. doi:10.1017/ S0007114507864841.

55. Briggs MA, Rumbold PLS, Cockburn E, Russell M, Stevenson EJ. Agreement between Two Methods of Dietary Data Collection in Male Adolescent Academy-Level Soccer Players. Nutrients Switzerland. 2015;7:5948-60. doi:10 3390/nu7075262.

56. Leblanc JC, Le Gall F, Grandjean V, Verger P. Nutritional intake of French soccer players at the clairefontaine training center. Int. J. Sport Nutr. Exerc. Metab. 2002;12:268-80.

57. Ziegler P, Sharp R, Hughes V, Evans W, Khoo CS. Nutritional status of teenage female competitive figure skaters. J Am Diet Assoc United States. 2002;102:374-9.

58. Aerenhouts D, Deriemaeker $P$, Hebbelinck M, Clarys P. Energy and macronutrient intake in adolescent sprint athletes: A follow-up study. J Sports Sci. 2011;29:73-82. doi:10.1080/02640414.2010.521946.

59. Kabasakalis A, Kalitsis K, Tsalis G, Mougios V. Imbalanced Nutrition of Top-Level Swimmers. Int J Sports Med. 2007:28:780-6. doi:10.1055/s-2007-964907.

60. Scaglioni S, Arrizza C, Vecchi F, Tedeschi S. Determinants of children ' s eating behavior. Am J Clin Nutr. 2011:94:S2006-11. doi:10.3945/ajcn.110.001685.1.

61. Pedersen S, Grønhøj A, Thøgersen J. Following family or friends. Social norms in adolescent healthy eating. Appetite. 2015;86:54-60. doi:10.1016/j. appet.2014.07.030

62. Spencer R, Rehman L, Kirk SF. Understanding gender norms, nutrition, and physical activity in adolescent girls: a scoping review. Int J Behav Nutr Phys Act. 2015;12:1-11. doi:10.1186/s12966-015-0166-8.

63. Deliens T, Deforche B, De Bourdeaudhuij I, Clarys P. Determinants of physical activity and sedentary behaviour in university students: a qualitative study using focus group discussions. BMC Public Health. 2015;15: 201-9. doi:10.1186/s12889-015-1553-4.

64. Sleddens E, Kroeze W, Kohl L, Bolten LM, Velema E, Kaspers PJ, et al. Determinants of dietary behavior among youth: an umbrella review. Int J Behav Nutr Phys Act. 2015;12:7. doi:10.1186/s12966-015-0164-X.

65. Sleddens EFC, Kroeze W, Kohl LFM, Bolten LM, Velema E, Kaspers P, et al. Correlates of dietary behavior in adults: an umbrella review. Nutr Rev. 2015; 73:477-99. doi:10.1093/nutrit/nuv007. 
66. Monteiro CA, Cannon G, Moubarac J-C, Martins APB, Martins CA, Garzillo J, et al. Dietary guidelines to nourish humanity and the planet in the twentyfirst century. A blueprint from Brazil. Public Health Nutr. 2015;18:2311-22. doi:10.1017/S1368980015002165.

67. Fardet A, Rock E, Bassama J, Bohuon P, Prabhasankar P, Monteiro C, et al. Current food classifications in epidemiological studies do not enable solid nutritional recommendations for preventing diet-related chronic diseases: the impact of food processing. Adv Nutr. 2015;6:629-38. doi:10.3945/an.115. 008789.

68. Moubarac J, Parra DC, Cannon G, Monteiro CA. Food Classification Systems Based on Food Processing: Significance and Implications for Policies and Actions: A Systematic Literature Review and Assessment. Curr Obes Rep. 2014;3:256-72. doi:10.1007/s13679-014-0092-0.

69. Monteiro CA, Moubarac J-C, Cannon G, Ng SW, Popkin B. Ultra-processed products are becoming dominant in the global food system. Obes Rev. 2013;14:21-8. doi:10.1111/obr.12107.

70. Akman M, Akan H, Izbirak G, Tanröver O, Tilev SM, Yildiz A, et al. Eating patterns of Turkish adolescents: A cross-sectional survey. Nutr J. 2010;9 doi:10.1186/1475-2891-9-67.

71. Erdman KA, Tunnicliffe J, Lun VM, Reimer RA. Eating patterns and composition of meals and snacks in elite Canadian athletes. Int J Sport Nutr Exerc Metab. 2013;23:210-9.

72. van Kleef E, Shimizu M, Wansink B. Food compensation: do exercise ads change food intake? Int J Behav Nutr Phys Act. 2011;8:6. doi:10.1186/14795868-8-6.

73. Holubcikova J, Kolarcik P, Madarasova Geckova A, van Dijk JP, Reijneveld SA Lack of parental rule-setting on eating is associated with a wide range of adolescent unhealthy eating behaviour both for boys and girls. BMC Public Health. 2016;16:359. doi:10.1186/s12889-016-3002-4.

74. Ward SA, Bélanger MF, Donovan D, Carrier N. Relationship between eating behaviors and physical activity of preschoolers and their peers: a systematic review. Int J Behav Nutr Phys Act. 2016;13:50. doi:10.1186/s12966-016-0374-x.

75. PAHO. Pan American Health Organization/ World Health Organization [Internet]. 2016 [cited 2017 Feb 5].

76. World Health Organization. Addressing and managing conflicts of interest: in the planning and delivery of nutrition programmes at country level. 2015.

77. Gedrich K. Determinants of nutritional behaviour: a multitude of levers for successful intervention? Appetite. 2003;41:231-8. doi:10.1016/j.appet.2003.08. 005.

78. Kelly B, Baur LA, Bauman AE, King L, Chapman K, Smith BJ. Examining opportunities for promotion of healthy eating at children's sports clubs. Aust N Z J Public Health. 2010;34:583-8. doi:10.1111/j.1753-6405.2010.00619.x.

79. Wolfenden L, Kingsland M, Rowland BC, Dodds P, Gillham K, Yoong SL, et al. Improving availability, promotion and purchase of fruit and vegetable and non sugar-sweetened drink products at community sporting clubs: a randomised trial. Int J Behav Nutr Phys Act. 2015;12:35. doi:10.1186/s12966015-0193-5

80. Hull MV, Jagim AR, Oliver JM, Greenwood M, Busteed DR, Jones MT. Gender differences and access to a sports dietitian influence dietary habits of collegiate athletes. J Int Soc Sports Nutr. 2016;13:38. doi:10.1186/s12970-0160149-4.

\section{Submit your next manuscript to BioMed Central and we will help you at every step:}

- We accept pre-submission inquiries

- Our selector tool helps you to find the most relevant journal

- We provide round the clock customer support

- Convenient online submission

- Thorough peer review

- Inclusion in PubMed and all major indexing services

- Maximum visibility for your research

Submit your manuscript at www.biomedcentral.com/submit 